\title{
ON THE ORIGIN OF HIGHEST ENERGY COSMIC RAYS
}

\author{
G. Sigl ${ }^{a, b}$, D. N. Schramm ${ }^{a, b}$ and P. Bhattacharjee ${ }^{c}$ \\ ${ }^{a}$ Department of Astronomy \& Astrophysics \\ Enrico Fermi Institute, The University of Chicago, Chicago, IL 60637-1433 \\ ${ }^{b}$ NASA/Fermilab Astrophysics Center \\ Fermi National Accelerator Laboratory, Batavia, IL 60510-0500 \\ ${ }^{c}$ Indian Institute of Astrophysics \\ Sarjapur Road, Koramangala, Bangalore 560 034, INDIA
}

\begin{abstract}
In this paper we show that the conventional diffusive shock acceleration mechanism for cosmic rays associated with relativistic astrophysical shocks in active galactic nuclei (AGNs) has severe difficulties to explain the highest energy cosmic ray events. We show that protons above around $2 \times 10^{20} \mathrm{eV}$ could have marginally been produced by this mechanism in an AGN or a rich galaxy cluster not further away than around $100 \mathrm{Mpc}$. However, for the highest energy Fly's Eye and Yakutsk events this is inconsistent with the observed arrival directions. Galactic and intergalactic magnetic fields appear unable to alter the direction of such energetic particles by more than a few degrees. We also discuss some other options for these events associated with relativistic particles including pulsar acceleration of high $Z$ nuclei. At the present stage of knowledge the concept of topological defects left over from the early universe as the source for such events appears to be a promising option. Such sources are discussed and possible tests of this hypothesis are proposed.
\end{abstract}

\footnotetext{
${ }^{1}$ Submitted to Astroparticle Physics
} 


\section{Introduction}

It is generally believed that cosmic rays with energies up to the "ankle" at around $3 \times 10^{18} \mathrm{eV}$ are predominantly of galactic origin [1] and that energies up to around $10^{14} \mathrm{eV}$ can be achieved by first order Fermi acceleration in shocks produced by supernovae exploding into the interstellar medium [2]. Recently the Fly's Eye detector revealed a change in the cosmic ray composition which is correlated with a dip in the total energy spectrum [1] located at the ankle. Around this dip the spectrum first steepens and then flattens again to a spectral index of around 2.7 which is even smaller than the index of 3 corresponding to the spectrum below the steepening. The data are consistent with a superposition of a steep power law spectrum of heavy nuclei and a flatter spectrum of protons which overtakes the former component at energies above the ankle. It is expected that this latter high energy proton component is of extragalactic origin. Furthermore, on 15 October 1991, the Fly's Eye observed an event at $(3.2 \pm 0.6) \times 10^{20} \mathrm{eV}(1 \sigma$ errors) [3], which is the event with the highest energy ever recorded. Interesting enough, the world's second highest energy air shower of $(1.1 \pm 0.4) \times 10^{20} \mathrm{eV}$ was recorded at Yakutsk [6, 7] located within 7.8 degrees from the Fly's Eye event (see Fig. 2). In this paper we will assume that these events were caused by relativistic particles.

There is plenty observational evidence that AGNs and radiogalaxies contain relativistic termination shocks which are likely to produce high energy cosmic rays. It therefore seems natural to extend the standard diffusive shock acceleration scenario which works well for supernova shocks at lower cosmic ray energies to larger extragalactic shock scales in order to explain the origin of this higher energetic extragalactic component. However, it turns out to be hard to explain the highest energy events by this mechanism. An interesting option is provided by decaying or annihilating topological defects which could be left over from phase transitions in the early universe at temperatures corresponding to some Grand Unification scale. The rest of the paper is organized as follows: We first reconsider in section 2 the source spectrum cutoff energy $E_{c}$ for shock accelerated cosmic rays as a function of shock size and average magnetic field strenght on this lenght scale and evaluate it for some typical observational numbers. In section 3 we discuss propagation effects on protons and show that at least the $3.2 \times 10^{20} \mathrm{eV}$ event is difficult to reconcile with the observational knowledge of typical extragalactic shock parameters in this acceleration scenario. We therefore discuss in section 4 some other options for these highest energy events. In section 5 we suggest that such events could alternatively be produced by topological defects. Finally we summarize our findings in section 6 .

\section{The Source Spectrum Cutoff for Extragalactic Shock Acceleration}

In relativistic shocks the cutoff energy $E_{c}$ for the source spectrum of accelerated cosmic rays is in the test particle approximation always given by $Z e B R$, the product of the charge $Z e$ of the cosmic ray particle, the magnetic field $B$ and the size $R$ of the shock, multiplied by 
some factor of order unity [9, 10] (we use natural units, i.e. $c=h / 2 \pi=1$, throughout this paper). However, it turns out that for the highest energies the mean free path of the particle becomes comparable to the shock size $R$, which sometimes is not properly accounted for. We therefore calculate here our own approximation for $E_{c}$.

\subsection{The Source Energy Cutoff}

The acceleration of a cosmic ray particle of energy $E$ in an astrophysical shock is governed by the equation

$$
\frac{d E}{d t}=\frac{E}{T_{a c c}}
$$

where $T_{a c c}$ is the energy dependent acceleration time. In the statistical point of view the slope $q$ of the energy spectrum $d N / d E \propto E^{-q}$ of the particle flux is related to $T_{a c c}$ and $T_{e s c}$, the mean (in general also energy dependent) escape time by [2, 9]

$$
q=1+\frac{T_{a c c}}{T_{e s c}}
$$

For first order Fermi acceleration at nonrelativistic shocks caused by supernovae, $T_{a c c}$ is usually given by

$$
T_{a c c}=\frac{3}{u_{1}-u_{2}}\left(\frac{D_{1}}{u_{1}}+\frac{D_{2}}{u_{2}}\right)
$$

where $u_{1}, u_{2}$ are the up- and downstream velocities of the shock and $D_{1}$ and $D_{2}$ are the corresponding diffusion coefficients, respectively. Diffusion is dominated by magnetic pitch angle scattering caused by inhomogeneities in the magnetic field [11, 12]. Therefore, the mean free path $\lambda$ is bounded from below by some multiple $g$ of the gyroradius $r_{L}=E /(Z e B)$ and $D_{1}$ and $D_{2}$ can for ultrarelativistic particles be estimated by

$$
D_{1}, D_{2} \sim \lambda / 3 \gtrsim \frac{g E}{3 Z e B} .
$$

For nonrelativistic shocks, $g$ is usually set equal to 1 [2, 9]. However, as we deal with the highest energetic extragalactic cosmic ray component, we have to consider relativistic shocks because they provide the most powerful accelerators. Monte-Carlo simulations of such relativistic shocks yield $g \sim 40$ [10, 13]. Furthermore, the acceleration turns out to be enhanced compared to eq. (3) by about a factor of 10 in highly inclined [13] and by about a factor of 13.5 in parallel [14] relativistic shocks, respectively.

Putting everything together and maximizing $T_{a c c}$ from eq. (3) we arrive at

$$
T_{a c c} \gtrsim \frac{g}{2.25} \frac{E}{Z e B},
$$

On the other hand, as long as the diffusion approximation is valid, i.e. as long as $\lambda<R$ corresponding to $E<E_{\text {diff }} \equiv Z e B R / b$, the escape time is given by $T_{e s c}=R^{2} / \lambda$, whereas for 
$E \geq E_{\text {diff }}$ the particles are freely streaming out of the shock region to a good approximation and $T_{e s c}=R$. Using eqs. (2) and (5), we thus get

$$
q\left(E>E_{d i f f}\right) \sim 1+\frac{E}{2.25 E_{\text {diff }}} .
$$

Defining the cutoff energy $E_{c}$ as the energy where the source spectral index becomes 3 (remember that the slope of the energy spectrum observed at the earth was around 2.7 in the region of highest energies) yields

$$
E_{c} \equiv E_{q=3} \sim 10^{17} \mathrm{eV} Z\left(\frac{R}{\mathrm{kpc}}\right)\left(\frac{B}{\mu \mathrm{G}}\right) .
$$

This is compatible or even higher as compared to similar estimates [9, 10, 15, 16, 17]. We have assumed here that the magnetic field is parallel to the shock normal. If that is not the case there will be an electric field $\mathbf{E}=\mathbf{u} \times \mathbf{B}$ in the shock rest frame ( $\mathbf{u}$ is the shock velocity in the lab frame). This causes drift acceleration of charged particles to a maximal energy given by

$$
E_{\text {max }}=Z e u B R \sim 10^{18} \mathrm{eV} Z u\left(\frac{R}{\mathrm{kpc}}\right)\left(\frac{B}{\mu \mathrm{G}}\right),
$$

which is around one order of magnitude larger than eq. (7) if $u$ approaches the speed of light. However, the electric field $\mathbf{E}$ is expected to be much smaller in general due to plasma effects so that rather special conditions have to be fulfilled in order that such high energies can be approached.

Throughout the rest of this section and the next section we will restrict our discussion to protons $(Z=1)$. We will comment on nuclei as possible candidates for events with energies above $10^{20} \mathrm{eV}$ in section 4.3 .

\subsection{The Cutoff in Numbers}

Let us now look at some observational numbers for $R$ and $B$ and evaluate the corresponding cutoff energy for a proton. Cesarsky [9] cited the example of a galaxy encounter (NGC $4038 / 39$ [18]) as a location of a strong relativistic shock with a magnetic field of about $40 \mu \mathrm{G}$ on a scale of about $2 \mathrm{kpc}$, leading to $E_{c} \sim 8 \times 10^{18} \mathrm{eV}$, significantly too small to explain the origin of cosmic rays with energies as high as the Fly's Eye event of $3 \times 10^{20} \mathrm{eV}$. Potentially more interesting candidates for acceleration beyond $10^{20} \mathrm{eV}$ are revealed by the "hot spots" and radio lobes of CygA with $B \sim 400 \mu \mathrm{G}, R \sim 3 \mathrm{kpc}$ and $B \sim 4 \mu \mathrm{G}, R \sim 300 \mathrm{kpc}$, respectively [19], cited by Quenby [10] which lead to a cutoff energy $E_{c} \lesssim 5 \times 10^{20} \mathrm{eV}$. There are also some indirect indications from gamma ray astronomy that in some quasars protons could be shock accelerated to energies of about $2 \times 10^{20} \mathrm{eV}$ [20].

There is one nearby object where pure application of formula (7) on observational indications for $R$ and $B$ leads to an $E_{c}$ larger than $3 \times 10^{20} \mathrm{eV}$. This is if one takes the whole Virgo cluster with an extension of $R \sim 10 \mathrm{Mpc}$ and a intracluster magnetic field $B \sim 1.5 \mu \mathrm{G}$ 
which is compatible with observations [9, 21] and leads to $E_{c} \sim 1.5 \times 10^{21} \mathrm{eV}$. However, it is highly improbable that the whole Virgo cluster moving through intercluster space forms a relativistic shock of such an enormous extension, effective in coherent cosmic ray particle acceleration.

Based on examples of the sort presented above there is some common belief that for protons the highest source energy achievable by diffusive shock acceleration in quasars and radiogalaxies is around $10^{21} \mathrm{eV}[16,[17,22]$. We will nevertheless show in the next section that even if this is true, it is still difficult to explain the observed Fly's Eye event because of the information we have on its arrival direction.

\section{Propagation Effects on Protons above $100 \mathrm{EeV}$}

\subsection{Overview}

Up to now we were only talking about the source energy spectrum. However, a proton traveling through space is in general subject to interactions, mainly with photons and magnetic fields. The latter effect leads to a curved path with a radius given by the gyroradius

$$
r_{L}=1.1 \mathrm{pc}\left(\frac{E}{10^{15} \mathrm{eV}}\right)\left(\frac{B}{\mu \mathrm{G}}\right)^{-1}
$$

The former effect leads to scattering and an effective energy loss as long as the proton energy lies above a kinematical threshold energy

$$
E_{t h}=\frac{W_{t h}^{2}-m_{N}^{2}}{2 \epsilon(1+\cos \theta)},
$$

depending on the angle $\theta$ between the incoming photon momentum and the negative nucleon momentum. Here $m_{N}$ is the nucleon mass, $\epsilon$ is the typical energy of an incoming photon and $W_{t h}$ is the center of mass energy threshold for the particular reaction under consideration. The most important ones are electron pair production $N+\gamma \rightarrow N+e^{+}+e^{-}$with $W_{t h}^{e^{+} e^{-}}=$ $m_{N}+2 m_{e}$, and pion production, $N+\gamma \rightarrow N+\pi$, with $W_{t h}^{\pi}=m_{N}+m_{\pi}$, with $m_{e}$ and $m_{\pi}$ the electron and pion mass, respectively. For a fraction of its propagation time depending

on the effective neutron lifetime the proton will actually transform into a neutron which does, however, not have much influence on this treatment as protons and neutrons have similar interactions with the MBR. Thus, nucleons interact with photons of the microwave background radiation (MBR) producing $e^{+} e^{-}$pairs and pions above an energy of about $5 \times 10^{17} \mathrm{eV}$ and $1.1 \times 10^{20} \mathrm{eV}$, respectively. The latter effect is known as the GreisenZatsepin-Kuz'min (GZK) effect [23]. There are also plenty of infrared and optical photons around luminous AGNs and galaxy clusters and especially in their central regions leading to correspondingly smaller nucleon threshold energies. However, their number density is in general not much bigger than that of the MBR photons and the cross section at the correspondingly higher center of mass energy is even smaller. This implies that possible 
interactions with these higher energy photons does not substantially reduce the mean free path below $6 \mathrm{Mpc}$, the typical mean free path in the MBR 24]. As this is much larger than typical galaxy sizes, we will restrict our considerations to interactions with the MBR. That means that we are on the safe side and get lower limits on energy losses and upper limits on the corresponding possible travel distances [25].

\subsection{Energy Losses}

We now ask the question in what distance range a source causing nucleon induced events of energy $E_{0}$ on earth could be if the maximal source energy is $E_{s}$. To this end we introduce the "longitudinal nucleon energy" $\tilde{E}_{N}=(1+\cos \theta) E_{N} / 2 \gtrsim E_{t h}$ besides the nucleon energy $E_{N}$ in the comoving frame. The last inequality holds because of eq. (10) as long as reactions are kinematically allowed. By performing a Lorentz transformation from the comoving frame to the center of mass frame corresponding to a gamma factor $\gamma_{c m} \sim \tilde{E}_{N} / W=\tilde{E}_{N} /\left(m_{N}^{2}+\right.$ $\left.4 \epsilon \tilde{E}_{N}\right)^{1 / 2}$ one can see that after scattering the nucleon energy in the comoving frame is given by

$$
E_{N}^{\prime} \sim \gamma_{c m}\left(E_{c m}^{\prime}+p_{c m}^{\prime} \cos \theta_{c m}\right) \sim \frac{\tilde{E}_{N}}{W}\left(E_{c m}^{\prime}+p_{c m}^{\prime} \cos \theta_{c m}\right)
$$

where $p_{c m}^{\prime}$ and $E_{c m}^{\prime}$ are momentum and energy of the nucleon after scattering and $\theta_{c m}$ is the scattering angle, evaluated in the center of mass frame, respectively. Above $10^{20} \mathrm{eV}$ the energy loss is dominated by pion production [26] for which these quantities are related to $W$ by $\left(m_{N}^{2}+p_{c m}^{\prime 2}\right)^{1 / 2}+\left(m_{\pi}^{2}+p_{c m}^{\prime 2}\right)^{1 / 2}=W$. As we are only interested in an estimate we set $\tilde{E}_{N}=E_{N} / 2$ and neglect the energy dependence of the mean free path (which in the energy range we are interested in is a good approximation [24]) $\lambda \sim 6 \mathrm{Mpc}$ in the following calculation. Because of eq. (11) the energy change in a scattering event $\xi\left(E, \theta_{c m}\right)$ relative to the lower energy is given by

$$
\xi\left(E, \theta_{c m}\right)=\frac{W}{E_{c m}^{\prime}+p_{c m}^{\prime} \cos \theta_{c m}}-1 .
$$

Furthermore, we neglect energy loss due to cosmological redshift as we are dealing with noncosmological distances at these high energies. By integrating from lower to higher energies one can show that the mean $\bar{l}$ and the variance $\Delta l^{2}$ of the distance as a function of $E_{0}$ and $E_{s}$ can be estimated by

$$
\bar{l}\left(E_{0}, E_{s}\right) \sim \lambda \int_{E_{0}}^{E_{s}} \frac{d E}{E \bar{\xi}(E)}, \quad \Delta l^{2}\left(E_{0}, E_{s}\right) \sim \lambda \bar{l}\left(E_{0}, E_{s}\right)+\frac{\lambda^{2}}{3} \int_{E_{0}}^{E_{s}} \frac{d E}{E \bar{\xi}^{3}(E)}\left(\frac{p_{c m}^{\prime}}{E_{c m}^{\prime}}\right)^{2} .
$$

The first term in the variance is due to the fluctuation of the number of scatterings and the second one is due to the fluctuation of the energy transfer $\xi$ of eq. (12) around its mean $\bar{\xi}(E) \sim W / E_{c m}^{\prime}-1$ averaged over the center of mass scattering angle $\theta_{c m}$. We have numerically integrated eq. (13). The results are shown in Fig. 1a and 1b. We see that for $E_{0}=1.7 \times 10^{20} \mathrm{eV}$, the lowest possible energy for the Fly's Eye event the distance must be 
smaller than $\sim 100 \mathrm{Mpc}$ and $\sim 130 \mathrm{Mpc}$ on the $3 \sigma$ level for a source energy $E_{s}=10^{21} \mathrm{eV}$ and $E_{s}=10^{22} \mathrm{eV}$, respectively. For the best fit energy of $3.2 \times 10^{20} \mathrm{eV}$ we get $\sim 60 \mathrm{Mpc}$ and $\sim 90 \mathrm{Mpc}$ for the corresponding $3 \sigma$ upper limits of distance for the same source energies.

Thus from the energy point of view an AGN or a galaxy cluster constituting a large scale shock with the intercluster medium could be marginally able to cause events like the $3 \times 10^{20} \mathrm{eV}$ Fly's Eye event by shock accelerated protons, if it is not much further away than $100 \mathrm{Mpc}$. Note that this number actually means the path length for which the distance is a lower limit which can be overtaken if the path is curved. However, we now show that the arrival direction of such a nucleon would then have to lie within about 10 degrees of the direction of its source if conventional wisdom about magnetic fields is used.

\subsection{Deviations from Rectilinear Propagation}

Let us first discuss deflection caused by magnetic fields. Unfortunately, not much is known about extragalactic magnetic fields. Faraday rotation measurements of extragalactic radio sources seem to suggest fields of the order of $10^{-9} \mathrm{G}$ which could be homogeneous on large scales [27]. Most estimates are of this order or below [28], a more recent one being as low as $3 \times$ $10^{-11} \mathrm{G}$ [29]. The bending angle $\alpha$ in radian for a proton traveling in a magnetic field satisfies $\alpha \lesssim \int d l / r_{L}$ where $d l$ is the differential path length and $r_{L}$ is given by eq. (9). A $10^{-9} \mathrm{G}$ field leads to a maximal bending angle of around 10 degrees for a proton with arrival energy of $3 \times 10^{20} \mathrm{eV}$. This maximum can only be reached if the magnetic field is perpendicular to the proton path and does not change its polarization considerably on a scale of $\sim 100 \mathrm{Mpc}$. Otherwise the bending angle is reduced at least by a factor $\left(d_{c} / 100 \mathrm{Mpc}\right)^{1 / 2}$ where $d_{c}$ is the magnetic field coherence length scale. Therefore, even if the typical intercluster field would be as high as $10^{-8} \mathrm{G}\left[30\right.$ the bending angle would still not be larger than $10^{\circ}$ if the coherence length scale is $\sim 1 \mathrm{Mpc}$.

A proton can also be deflected by our own galactic magnetic field which is of the order of $3 \mu \mathrm{G}$ [27, 31]. Based on radio telescope observations of Faraday rotations [31] its coherent component is supposed to have a cylindrical structure of diameter $\sim 25 \mathrm{kpc}$ and height of order $1 \mathrm{kpc}$ and being polarized in the direction of decreasing galactic longitude in the outer region. The random component is supposed to be of the same order of magnitude. Given the arrival direction of the Fly's Eye and the Yakutsk events shown in Fig. 2 we see that the corresponding path length through the field is less than $3 \mathrm{kpc}$ resulting in a maximum bending angle of $\sim 2^{\circ}$.

We finally remark that even though magnetic fields in galaxy clusters as high as $10^{-6} \mathrm{G}$ as already mentioned [21] could cause a significant deflection this does not influence our argumentation. It only means that if our proton encountered such "magnetic lenses" it should point approximately to the last encountered. There still has to be a nearby galaxy cluster in its arrival direction. Furthermore, the above mentioned fact that a proton partly transforms into a neutron during propagation even tends to decrease the bending further.

The propagation direction could in principle also be changed by scattering with photons, but the scattering angles involved are much too small. To see that we use our notation from 
above and note that the scattering angle $\theta_{s c}$ in the comoving frame obeys

$$
\tan \theta_{s c} \lesssim \frac{p_{c m}^{\prime} \sin \theta_{c m}}{\gamma_{c m}\left(p_{c m}^{\prime} \cos \theta_{c m}+E_{c m}^{\prime}\right)} .
$$

Maximizing with respect to $\theta_{c m}$ and using $p_{c m}^{\prime} \leq\left(W^{2}-m_{N}^{2}\right)^{1 / 2} \leq\left(4 \epsilon \tilde{E}_{N}\right)^{1 / 2}$ leads to an estimate independent from the final state $X$ in the reaction $N+\gamma \rightarrow N+X$,

$$
\tan \theta_{s c} \lesssim\left[\frac{4 \epsilon}{\tilde{E}_{N}}\left(1+\frac{4 \epsilon \tilde{E}_{N}}{m_{N}^{2}}\right)\right]^{1 / 2} \lesssim 10^{-11}
$$

where we have used $\epsilon \sim 3 \times 10^{-4} \mathrm{eV}$ and $\tilde{E}_{N} \gtrsim 3 \times 10^{20} \mathrm{eV}$. Because the mean free path at these energies is of order $6 \mathrm{Mpc}$ [24] we expect only a few scattering events during traveling over a distance of $100 \mathrm{Mpc}$ which thus never can lead to a significant change in the propagation direction.

The arrival direction of the Fly's Eye event is given by $\alpha=85.2^{\circ} \pm 1^{\circ}$ and $\delta=48^{\circ} \pm 10^{\circ}$. No potentially interesting object with a sufficiently powerful shock acceleration engine of the scale discussed in the previous section is located within $100 \mathrm{Mpc}$ in that direction. This can be seen from Fig. 2 where we show the directions to important nearby galactic objects, galaxy clusters and AGNs. One of the three prominent FR-II radio galaxies listed in [17] and thought to contribute to the proton spectrum between $10^{18} \mathrm{eV}$ and $10^{20} \mathrm{eV}$ (i.e. below the GZK threshold), namely 3C111, has the coordinates $\alpha=63.75^{\circ}, \delta=37.9^{\circ}$ and lies thus $(18.6 \pm 5.1)^{\circ}$ away from that direction. But it is at least $140 \mathrm{Mpc}$ away. The only quasars within around $10^{\circ}$ of the arrival direction are $3 \mathrm{C} 147$ and $3 \mathrm{C} 159$ [32], both of them at a distance of at least $900 \mathrm{Mpc}$. The only Seyfert galaxy within $10^{\circ}$ is MCG 08.11 at an angular distance of $(2.35 \pm 8.4)^{\circ}$ and a distance of at least $60 \mathrm{Mpc}$ [32]. Although this is much nearer than the before mentioned quasars it produces a much lower radio flux at the earth and therefore seems also not likely to produce high cosmic ray fluxes. Under the quite improbable assumptions discussed at the end of the previous section the Virgo cluster seems to be the nearest possible candidate. However, it is located around $90^{\circ}$ away from that direction.

\section{Other Options for Ultrahigh Energy Events}

We saw in the previous section that especially the Fly's Eye event is difficult to explain as a proton within the standard shock acceleration scenario. We therefore now like to discuss some other options beginning with secondary particles produced by shock accelerated protons.

\subsection{Secondary Photons}

Is the shower development of the highest energy Fly's Eye event consistent with what would be expected if it was caused by a photon? At these high energies the photon begins to 
interact with the earth's magnetic field already above the atmosphere. Fitting the shower shape with a three parameter Gaisser-Hillas shower development function [33 gives a depth of first interaction of $-100 \mathrm{~g} \mathrm{~cm}^{-2}$ and thus seems to indicate a first interaction above the atmosphere. However, it could also indicate that the fitting function is simply inappropriate at these high energies. Indeed, it is now believed that fitting heavy nuclei induced showers can lead to similar negative values [3]. Taking the LPM effect into account the average shower maximum is expected to be somewhat larger than for a proton induced shower with an average width about twice as large as the corresponding proton profile width [34]. As the fluctuations are expected to be large this could still be compatible with the reported maximum at $(815 \pm 50) \mathrm{g} \mathrm{cm}^{-2}$.

Photons of such a high energy have a secondary origin in the standard scenario as they are produced by decay of pions or $e^{+} e^{-}$interactions which in turn are produced by the interactions of the cosmic ray protons with the MBR. The photon mean free path becomes comparable with or larger than that of the protons above a few $10^{19} \mathrm{eV}$ [35]. The exact value of the photon to proton ratio depends on the universal radio background and the intergalactic magnetic field strength. The former leads to additional losses due to electron pair production. The latter leads to an inefficient electromagnetic cascade development $\gamma+\gamma_{b} \rightarrow e^{+} e^{-}, e^{+} e^{-}+\gamma_{b} \rightarrow e^{+} e^{-}+\gamma\left(\gamma_{b}\right.$ is the background photon) due to synchrotron cooling of the electrons even for fields as low as $10^{-10} \mathrm{G}$. Typical estimates for the photon to proton ratios are considerably smaller than 1 above $10^{20} \mathrm{eV}$ [36, 37. That makes the problem even harder as there have to be (even more abundant and of higher energy) primary protons acting as the source for such photons. Wolfendale [22 claims that the photon to proton ratio could exceed unity above $\sim 3 \times 10^{20} \mathrm{eV}$ if the source energy cutoff $E_{c}$ is much above $10^{21} \mathrm{eV}$. This possibility thus runs into trouble with our discussion of $E_{c}$ in section 2 .

\subsection{Secondary Neutrinos}

Could the highest energy Fly's Eye event have been an extragalactic neutrino produced as a secondary of a shock accelerated proton? Because neutrinos essentially lose no energy apart from redshift in going over cosmological distances it could have been produced by a proton interacting near its acceleration site thus avoiding excessive subsequent energy losses due to downscattering. However, it turns out that the neutrino yield above $10^{18} \mathrm{eV}$ is considerably smaller than one for all reasonable injection spectra. Furthermore, at the highest energies the spectral index observed at the earth is predicted to be 0.5 larger than the corresponding

proton spectral index [38]. As even at these energies the neutrino nucleon cross section is still by a factor of at least $10^{6}$ smaller than the nucleon nucleon (and also the gamma nucleon) cross section the average interaction depth is much larger than the atmospheric depth. All that leads to the conclusion that the event rate due to neutrinos should be much smaller than that due to protons at the same energy. One would also expect the neutrino induced showers to start predominantly at high depths i.e. near the horizon [36]. Therefore, if a neutrino caused the event then it was a very atypical one and we would expect much more proton and even photon events at the same energy. 


\subsection{Secondary Neutrons and Heavy Nuclei}

In the shock acceleration scenario neutrons are also produced as secondaries of protons or heavy nuclei as they are neutral and can not be accelerated directly. Furthermore, due to instability they have only a finite range which for $3 \times 10^{20} \mathrm{eV}$ is about $3 \mathrm{Mpc}$. The puzzle is therefore not solved if our events are caused by neutrons.

Looking at eqs. (7) and (8) one realizes that heavy nuclei can reach maximal energies which are higher by a factor $Z$ compared to the protons. However, heavy nuclei lose energy not only due to the processes which dominate the energy loss of protons but also due to the giant dipole resonance which leads to photodisintegration. Above $10^{20} \mathrm{eV}$ the corresponding energy loss rates are about a factor 10 higher than those for protons [26] and are typically due to proton stripping reactions. For example, a ${ }^{56} \mathrm{Fe}$ nucleus being launched with an energy of $10^{21} \mathrm{eV}$ will be below $10^{20} \mathrm{eV}$ after traveling $8 \mathrm{Mpc}$ [24]. Thus, for our purposes nuclei are only interesting when they are of galactic origin. There are two galactic sites which could provide acceleration to interesting energies for heavy nuclei. The first one is the termination shock of the galactic wind caused by the milky way [39]. This leads to maximal energies of $\sim 3 \times 10^{17} \mathrm{eV} Z\left(u / 600 \mathrm{kms}^{-1}\right)^{2}\left(T_{A} / 1.5 \times 10^{10} \mathrm{y}\right)(B / 0.1 \mu \mathrm{G})$ [2] where $u_{w}$ is the galactic wind speed and $T_{A}$ is the shock lifetime. Even for $Z \sim 100$ this is significantly too low and one is forced to use quite extreme parameters to reach beyond $10^{20} \mathrm{eV}$. The second site would be even more natural to produce predominantly high energy heavy nuclei, namely young supernova remnants which form a pulsar wind shock [2]. This is because pulsars can have quite large surface magnetic fields of order $10^{12} \mathrm{G}$ leading to fields of order $10 \mathrm{G}$ on scales of $10^{14} \mathrm{~cm}$ [2]. The pulsar wind can be relativistic so that application of eq. (8) leads to $E_{\max } \sim 10^{18} \mathrm{eV} Z$ which for $Z \sim 50$ is about one order of magnitude smaller than the highest observed energies. Adopting the more conservative estimate eq. (7) leads to a short fall of about two orders of magnitude.

Indeed, as can be seen from Fig. 2, the Crab nebula lies near the arrival direction of our events and is expected to have magnetic fields of the order of $10^{-3} \mathrm{G}$ on a scale of $2 \mathrm{pc}$ [10] leading to estimates for the energy cutoff quite similar to the above mentioned. However, a calculation including the uncertainty of the latter one gives a relative angle of $(25.9 \pm 7.5)^{\circ}$ which is more than $3 \sigma$ away. Furthermore, taking into account deflection effects due to the coherent galactic magnetic field component mentioned in section 3.3 increases the angular distance as the path should be bent towards the galactic north pole (see Fig. 2). For example, for $Z=5$ the angular difference from the arrival direction would be $(29.5 \pm 6.9)^{\circ}$. It thus seems that only large bending by almost $360^{\circ}$ could explain a possible origin from the Crab. For $Z \gtrsim 50$ the Larmor radius is $\lesssim 2 \mathrm{kpc}$ at these energies which indeed comes near the required amount of bending. In that case, however, the arrival direction is not expected to be correlated with the source location in a simple way and the propagation of these particles should better be considered as diffusion in the magnetic field. The source could therefore be any galactic site being able to produce the required source energy and this possibility can not be completely excluded although, as mentioned above, current models fall short in energy. It should be noted that the shower maximum at $(815 \pm 50) \mathrm{g} \mathrm{cm}^{-2}$ allows no definite distinction between a proton and a heavy nucleus induced shower as the expected numbers 
for these options are $\sim 850 \mathrm{~g} \mathrm{~cm}^{-2}$ and $\sim 775 \mathrm{~g} \mathrm{~cm}^{-2}$ for iron, respectively [2, 3].

There are still problems left in interpreting the $3 \times 10^{20} \mathrm{eV}$ event as caused by a heavy nucleus. As so it is possible that heavy nuclei could be disintegrated already at the source of acceleration [25]. Furthermore, the Fly's Eye data between $10^{18} \mathrm{eV}$ and $10^{20} \mathrm{eV}$ suggest the transition to a lighter component as we already mentioned.

\subsection{Some Other Options}

There were some other suggestions how one could get to higher source energies. For example, Colgate 41] claimed that in the relativistic plasma of AGN jets energies as high as $10^{24} \mathrm{eV}$ could be reached due to a plasma pinch effect similar than that used in Tokamaks. Note however that due to Fig. 1a and $1 \mathrm{~b}$ the source of the highest energy events could still not be much further away than $150 \mathrm{Mpc}$. Because the Larmor radius grows with energy the possible bending angle caused by magnetic fields could also not be enhanced significantly beyond 15 degrees so the problem remains.

It was suggested [42] that high energy events could be caused by relativistic dust grains. The lateral shower profile caused by a dust grain entering the atmosphere is expected not to show a broad maximum but instead to have a more or less constant lateral spread as long as the grain remains large and energetic enough to produce secondary particles. However, the highest energy Fly's Eye event showed a quite "normal" shower development typical for a primary proton or possibly a photon which leaves the dust grain hypothesis to seem not very likely. Furthermore there is a tendency for these grains to break up by interactions with photons and gas atoms in the interstellar medium [43].

\section{Cosmic Rays Produced by Topological Defects}

Topological defects (TDs) 44 could have been formed in the early universe during phase transitions associated with spontaneous breaking of symmetries implemented in unified models of high energy interactions. Such TDs are magnetic monopoles, cosmic strings, domain walls, superconducting cosmic strings, textures, etc. TDs are topologically stable but can nevertheless be destroyed due to physical processes like collapse or annihilation 45, 46, 47, 48]. In that case the energy stored in the defects is released in the form of massive quanta of the fields like gauge fields and Higgs fields associated with the broken symmetry. These "X" particles released from the TDs would typically decay into quarks and leptons. Hadronization of the quarks would produce jets of hadrons containing mainly light mesons (pions) together with a small fraction $(\sim 3 \%)$ of nucleons. The gamma rays and neutrinos from the decay of the pions would thus be the dominant particles in the final decay products of the $\mathrm{X}$ particles. The mass $m_{X}$ of the $\mathrm{X}$ particles is typically of the order of the symmetry-breaking scale which in Grand Unified Theories (GUTs) can be $\sim 10^{25} \mathrm{eV}$, or even the Planck scale $\sim 10^{28} \mathrm{eV}$. The decay of the $\mathrm{X}$ particles released from TDs can thus give rise to nucleons, gamma rays and neutrinos with energies up to $\sim m_{X}$, very much higher than what can be achieved by astrophysical shock acceleration mechanism. The cosmic ray 
particles can thus be produced directly in this scenario, and no acceleration mechanism is needed.

The production spectra of the nucleons, gamma rays and neutrinos in the TD scenario are determined by the physics of fragmentation of quarks into hadrons. Extrapolation 45 of QCD based hadronization models (which describe well the GeV scale collider data) to the extremely high energies gives a power-law approximation [47, 48, 49] to the differential production spectra with a power-law index $q \sim 1.32$ for nucleons as well as pions. The decay of the neutral pions thus gives a differential gamma ray production spectrum also with $q=1.32$. It is to be emphasized, however, that there is a great deal of uncertainty in extrapolating the low energy QCD models of hadronization to the extremely high energies involved in the present situation. Moreover, the gamma ray production spectrum can be somewhat different from the proton production spectrum if one considers the gamma rays generated by the charged leptons (electrons and positrons) in the primary decay products of the $\mathrm{X}$ particles. The electrons and positrons coming from the decay of the charged pions in the hadronic jets also contribute to the overall primary gamma rays. The main point, however, is that the production spectra of cosmic ray particles in the TD scenario can in principle be considerably flatter than in the standard shock acceleration scenario. The latter, to recall, by and large produces differential production spectra with $q>2$.

One consequence of a relatively flat production spectrum in the TD scenario would be the "recovery" [4] of the evolved proton spectrum after the GZK "cutoff" [23]. While this is heartening from the point of view of prospects for detecting protons above the GZK "cutoff", too flat a proton spectrum may cause problems in that it may give rise to excessive gamma ray flux at much lower energies, as discussed below. In any case, as first discussed in Ref. [37], the photon-to-proton ratio in the evolved spectra can be considerably larger than 1 above $10^{20} \mathrm{eV}$ in the TD scenario [37] (because of the primary gamma rays which outnumber the protons by a factor of at least 10 at production, and also because of higher transparency of gamma rays relative to the protons at these energies), and so the cosmic rays above $10^{20} \mathrm{eV}$ are predicted to be mainly primary gamma rays rather than protons.

Gamma rays as well as protons of ultrahigh energies generate lower energy gamma rays by $\gamma-\gamma_{b}$ and $p-\gamma_{b}$ collisions with the photons $\left(\gamma_{b}\right)$ of the background radiation fields. The electromagnetic component of the energy lost by the photons and protons in these collisions cascades down to lower energies by electromagnetic cascading in the universal radio background (URB), the microwave background (CMBR), and in the infrared background (IRB) (in order of decreasing energy of the propagating photon). Recently it has been realised [50, 51, 52] that the measured flux of extragalactic gamma rays in the $100 \mathrm{MeV}$ region 53 provides an upper limit on the total energy density of the cascade-initiating electromagnetic radiation that can possibly be released in the universe due to $p-\gamma_{b}$ and $\gamma-\gamma_{b}$ interactions. This, in turn, restricts the shapes of the proton as well as the primary photon spectra in the highest energy region. The authors of Ref. [50] claim that a proton spectrum with $q=1.32$ at injection and extending to $10^{24} \mathrm{eV}$ would by itself give rise to a $100 \mathrm{MeV}$ gamma ray flux exceeding the measured flux by a factor of 2 if the evolved proton spectrum is normalized [37, 49] to observed particle flux at $4 \times 10^{19} \mathrm{eV}$. This question has 
recently been studied in detail [52] by a careful numerical calculation of the cascading process including the gamma rays generated by both $p-\gamma_{b}$ as well as the $\gamma-\gamma_{b}$ processes. It is found that whether or not the predicted $100 \mathrm{MeV}$ gamma ray flux exceeds the measured value depends strongly on the level of the IRB as well as on its cosmological evolutionary history both of which are rather uncertain, and so a firm conclusion in this regard cannot be drawn at this stage. Nevertheless, the authors of Ref. [51 have suggested that the possible problem arising from requirement of consistency with the measured $100 \mathrm{MeV}$ gamma rays can be avoided if the cosmic rays above $10^{19} \mathrm{eV}$ are mainly gamma rays and not protons. The preliminary analysis of Ref. [51] shows that this is possible provided the injection spectrum of the primary gamma rays above $10^{20} \mathrm{eV}$ in the TD model is made somewhat steeper $(q \sim 2.4)$ compared to the protons $(q \sim 1.5)$ and $\gamma / p$ is demanded to be $\sim 60$ at injection so that the proton component is made negligible compared to the photons. The average multiplicity in the hadronic jets arising out of the decay of the $\mathrm{X}$ particles is also required to be somewhat higher than what naive extrapolation of the low energy QCD based models of jet fragmentation indicates. While all these phenomenological requirements need to be substantiated on more theoretical grounds, the general conclusion that seems to arise from the above discussion is that the highest energy cosmic ray particles in the TD scenario should be mainly gamma rays and not protons. And, of course, primary neutrinos [49] should be at least as abundant as the gamma rays, perhaps even more.

Could the $3 \times 10^{20} \mathrm{eV}$ Fly's Eye event be a primary gamma ray due to TD collapse or annihilation? As already mentioned in Section 4.1 above, the shower development is not in contradiction with what is expected for a primary gamma. The resulting electromagnetic shower can in fact be very similar [54] to a proton-induced shower, although some differential parameters, e.g., muon/electron ratio at large distances from the core of the shower can in principle be used for effective separation [54] of these photon-induced showers from the proton-induced ones.

How could one distinguish between the TD option and the galactic heavy nuclei hypothesis which seems to be the least problematic option within the standard picture? Heavy nuclei are expected to produce substantially more muons compared to gammas of the same energy [2]. It should therefore also be possible to draw a decision between these options as more statistics is available at these highest energies.

The lack of any obviously identifiable astrophysical source for the event is not a problem for the TD scenario because TDs are not necessarily expected to be associated with any astrophysical sources such as galaxy clusters or AGNs. The TD model thus seems to offer an attractive option in this regard.

It is, however, expected that the same TD annihilation event would also produce lower energy gamma rays which would arrive at earth at roughly the same time and with same arrival direction as the $3 \times 10^{20} \mathrm{eV}$ Fly's Eye event. Unfortunately, the CASA array 555 capable of detecting such gamma rays was not operating at the time when the above Fly's Eye event was recorded. However, the CYGNUS array [56] capable of detecting gamma rays above about $100 \mathrm{TeV}$ was operating and it detected no event [57] that can be associated with the Fly's Eye event. If the integral primary gamma ray spectrum between $10^{14} \mathrm{eV}$ and 
$10^{20} \mathrm{eV}$ due to TD annihilation is taken to be approximately proportional to $E^{-\alpha},(\alpha>0)$, and if one (optimistically) takes the flux at $10^{20} \mathrm{eV}$ as $\sim 1$ per $1000 \mathrm{~km}^{2}$, then above $10^{14} \mathrm{eV}$ and in an area of $0.02 \mathrm{~km}^{2}$ (roughly the area of the CYGNUS array) one would expect an integral flux $F\left(E>10^{14} \mathrm{eV}\right) \sim 2 \times 10^{6 \alpha-5}$ events per $0.02 \mathrm{~km}^{2}$. The non-detection by the CYGNUS array of any gamma ray event in the $100 \mathrm{TeV}$ region coincident with the Fly's Eye event can then be interpreted in terms $\alpha$ being $\lesssim 0.78$ (i.e., a relatively flat spectrum) in the TD model at energies below $10^{20} \mathrm{eV}$. (Note that in conventional scenarios $\alpha$ is usually taken to be $\sim 1$ ). For example, if in the TD model one takes $\alpha \sim 0.32$ 49] and neglects the attenuation due to interaction with the CMBR (thus overestimating the expected flux), then $F\left(E>10^{14} \mathrm{eV}\right) \sim 0.0017$ events in $0.02 \mathrm{~km}^{2}$, and so CYGNUS may have missed the event. This point, however, needs further investigation, and will be discussed elsewhere.

\section{Conclusions}

We are lead to the conclusion that protons arriving at the earth with energies of $3 \times 10^{20} \mathrm{eV}$ or above are very likely to have come from an AGN or a galaxy cluster not further away than $100 \mathrm{Mpc}$ if they were produced there via the standard diffusive shock acceleration mechanism. Even then the necessary conditions to be fulfilled in such relativistic strong shocks seem highly improbable as long as the shock parameters have to be compatible with observational data. Furthermore, the arrival direction of such protons have to be within around 15 degrees in the direction of their source. The $3 \times 10^{20} \mathrm{eV}$ Fly's Eye event and the highest energy Yakutsk event were therefore very likely not protons produced within the standard diffusive shock acceleration scenario as they do not point to some possible source being nearer than $100 \mathrm{Mpc}$.

Some other explanations for such events like that being produced by secondaries of shock accelerated protons were discussed. Within the astrophysical shock scenario the most promising, although also problematic option seem to be heavy nuclei of galactic origin which could be accelerated in pulsar wind shocks driven by young supernova remnants.

We therefore conclude that at least some improvements in the understanding of the current acceleration picture have to be made in order to explain the highest energy cosmic rays observed. It seems possible that a completely new production mechanism for such particles is necessary. We suggested that the TD model could be a promising option. It is curious that such an exotic option seems to have less difficulties in explaining these ultrahigh energy particles. All other current options appear to require suspension of belief in seemingly well substantiated observational numbers or indicate incomplete understanding of the underlying physical process. We list all the options discussed here together with their problems in Table 1.

\section{Acknowledgements}

We would like to thank James Cronin, Eugene Parker, Hongyue Dai, Stirling Colgate, Gene Loh, Cy Hoffman and Corbin Covault for highly valuable discussions. In addition, we 
are grateful to Paul Sommers and Rene Ong for their suggestions concerning the manuscript. We would also like to thank Chris Hill for his collaboration in earlier papers dealing with topological defects and his enthusiastic contributions to this topic. This work has been supported, in part, by NSF, NASA and the DOE at the University of Chicago, by the DOE and by NASA through grant NAGW 2381 at Fermilab and by the Alexander-von-Humboldt foundation.

Note added: During preparation of this manuscript we became aware of a paper by P. Sommers [58] which essentially deals with the same topic. The conclusions reached concerning the shock acceleration scenario are quite similar. In difference, however, the aim of our paper was a more detailed discussion of all possible explanations related to relativistic particles and especially to note the emergence of the TD scenario as an interesting option.

\section{References}

[1] D. J. Bird et. al., Phys. Rev. Lett. 71, 3401 (1993).

[2] T. K. Gaisser: Cosmic Rays and Particle Physics, Cambridge 1990.

[3] We are grateful to Paul Sommers for providing us with these numbers and a discussion of it.

[4] Astrophysical Aspects of the Most Energetic Cosmic Rays, eds.: M. Nagano and F. Takahara, World Scientific, Singapore (1991).

[5] Proceedings of the Tokyo Workshop on Techniques for the Study of Extremely High Energy Cosmic Rays, published by the Institute for Cosmic Ray Research, University of Tokyo, ed.: M. Nagano (September 1993).

[6] N. N. Efimov et. al. in Ref. [4], p. 20.

[7] T. A. Egorov in Ref. [5], p. 35.

[8] Nucl. Phys. B (Proc. Suppl.) 28B (1992).

[9] C. T. Cesarsky in Ref. [8], p. 51.

[10] J. J. Quenby and K. Naidu in Ref. [8], p. 85.

[11] R. Blandford and D. Eichler, Physics Reports 154, 1 (1987).

[12] P. O. Lagage and C. J. Cesarsky, Astron. Astrophys. 125, 249 (1983).

[13] R. Lieu et.al., EUV publication no.552 (1993), to be published in Ap. J..

[14] J. J. Quenby and R. Lieu, Nature 342, 625 (1989). 
[15] A. M. Hillas, Ann. Rev. Astron. Astro. 22, 425 (1984).

[16] P. L. Biermann and P. A. Strittmatter, Ap. J. 322, 643 (1987).

[17] J. P. Rachen and P. L. Biermann, Astron. 83 Astroph. 272, 161 (1993).

[18] E. Hummel and J. M. van der Hulst, Astron. Astrophys. 155, 151 (1986).

[19] R. A. Liang, MNRAS 195, 261 (1981); P. J. Hargrave and M. Ryle, MNRAS 175, 481 (1976).

[20] K. Mannheim, Phys. Rev. D48, 2408 (1993).

[21] J. P. Vallee, Astron J. 99, 459 (1990).

[22] J. Wdowczyk and A. W. Wolfendale, Ap. J. 349, 35 (1990).

[23] K. Greisen, Phys. Rev. Lett. 16, 748 (1966); G. T. Zatsepin and V. A. Kuz'min, Pis'ma Zh. Eksp. Teor. Fiz. 4, 114 (1966) [JETP Lett. 4, 78 (1966)].

[24] J. W. Cronin, Nucl. Phys. (Proc. Suppl.) 28B, 213 (1992); private communications.

[25] J. B. Pollak and B. S. P. Shen, Phys. Rev. Lett. 23, 1358 (1969).

[26] J. L. Puget, F. W. Stecker and J. H. Bredekamp, Ap. J. 205, 638 (1976).

[27] E. N. Parker: Cosmical Magnetic Fields, Clarendon, Oxford (1979) and references therein.

[28] C. Heiles, Ann. Rev. Astron. Astro. 14, 1 (1976); G. L. Verschuur, Fund. Cosmic Phys. 5, 113 (1979); J. P. Vallee, Astrophys. Lett. 23, 85 (1983).

[29] E. Asseo and H. Sol, Phys. Rep. 48, 309 (1987).

[30] J. P. Vallee, Ap. J. 360, 1 (1990).

[31] J. P. Vallee, Ap. J. 366, 450 (1991).

[32] M. P. Veron-Cetty and P. Veron, A Catalogue of Quasars and Active Nuclei (ESO Report No. 13, November 1993).

[33] T. K. Gaisser and A. M. Hillas, Proc. of 15th ICRC 8, 353 (1977).

[34] L. G. Dedenko and I. M. Zheleznykh, Proc. of 21st ICRC 4, 1472 (1989).

[35] J. Wdowczyk, W. Tkaczyk and A. W. Wolfendale, J. Phys. A5, 1419 (1972); J. Wdowczyk, W. Tkaczyk, C. Adcock and A. W. Wolfendale, J. Phys. A4, L37 (1971); F. W. Stecker, Astrophys. Space Sci. 20, 47 (1973). 
[36] F. W. Stecker, Comments Astrophys. 7, 129 (1978); Astrophys. 228, 919 (1979).

[37] F. A. Aharonian, P. Bhattacharjee and D. N. Schramm, Phys. Rev. D46, 4188 (1992).

[38] C. T. Hill and D. N. Schramm, Phys. Rev. D31, 564 (1985).

[39] J. R. Jokipii and G. E. Morfill, Ap. J. 312, 170 (1987) and 290, L1 (1985).

[40] R. T. Emmering and R. A. Chevalier, Ap. J. 321, 334 (1987).

[41] S. Colgate, private communication.

[42] S. Hayakawa, Astrophys. Space Sci. 16, 238 (1972).

[43] V. S. Berezinsky and O. F. Prilutzky, Astrophys. Space Sci. 21, 475 (1973).

[44] For a review, see e.g. A. Vilenkin, Phys. Rep. 121, 263 (1985).

[45] C. T. Hill, Nucl. Phys B224, 469 (1983).

[46] C. T. Hill, D. N. Schramm and T. P. Walker, Phys. Rev. D36, 1007 (1987).

[47] P. Bhattacharjee and N. C. Rana, Phys. Lett. B 246, 365 (1990).

[48] P. Bhattacharjee, in Ref 四, pp. 382-399.

[49] P. Bhattacharjee, C. T. Hill and D. N. Schramm, Phys. Rev. Lett. 69, 567 (1992).

[50] X. Chi, C. Dahanayake, J. Wdowczyk and A. W. Wolfendale, Astropart. Phys. 1, 129 (1992).

[51] X. Chi, C. Dahanayake, J. Wdowczyk and A. W. Wolfendale, Astropart. Phys. 1, 239 (1993).

[52] F. Aharonian, P. Bhattacharjee, P. Coppi and D. N. Schramm, in preparation.

[53] C. E. Fichtel, G. A. Simpson, and D. J. Thompson, Ap. J. 222, 833 (1978).

[54] F. A. Aharonian, B. L. Kanevsky, and V. A. Sahakian, J. Phys. G. 17, 1909 (1991).

[55] R. A. Ong, Nucl. Phys. B (Proc. Suppl.) 14A, 273 (1990); J. W. Cronin et. al., EFI 94-05, submitted to Nucl. Inst. Meth.

[56] D. E. Alexandreas et. al., Nucl. Inst. Meth. A311, 350 (1992).

[57] C. Hoffmann, private communications.

[58] P. Sommers in Ref. [5], p. 23.

[59] We are grateful to Corbin Covault for helping us with this figure. 


\begin{tabular}{|c|c|c|c|}
\hline \multicolumn{4}{|c|}{ protons and secondary $\gamma^{\prime}$ s and $\nu$ 's } \\
\hline & \multicolumn{2}{|c|}{ maximal energy } & additional \\
\hline Sources & \multicolumn{2}{|l|}{ shock acc. } & problems \\
\hline AGNs & $10^{20} \mathrm{eV}\left(\frac{R B}{10^{3} \mathrm{kpc} \mu \mathrm{G}}\right)$ & $10^{21} \mathrm{eV}\left(\frac{R B}{10^{3} \mathrm{kpc} \mu \mathrm{G}}\right)$ & distance+direction \\
\hline pulsars & $2 \times 10^{17} \mathrm{eV}\left(\frac{R B}{0.002 \mathrm{pc} \mathrm{G}}\right)$ & $2 \times 10^{18} \mathrm{eV}\left(\frac{R B}{0.002 \mathrm{pc} \mathrm{G}}\right)$ & direction \\
\hline galactic wind & \multicolumn{2}{|c|}{$3 \times 10^{17} \mathrm{eV}\left(\frac{u}{600 \mathrm{kms}^{-1}}\right)^{2}\left(\frac{T_{A}}{1.5 \times 10^{10} \mathrm{y}}\right)\left(\frac{B}{0.1 \mu \mathrm{G}}\right)$} & \\
\hline \multicolumn{4}{|c|}{ galactic heavy nuclei } \\
\hline \multirow[b]{2}{*}{ Sources } & \multicolumn{2}{|c|}{ maximal energy } & additional \\
\hline & shock acc. & drift acc. & problems \\
\hline pulsars & $2 \times 10^{17} \mathrm{eV} Z\left(\frac{R B}{0.002 \mathrm{pc} \mathrm{G}}\right)$ & $2 \times 10^{18} \mathrm{eV} Z\left(\frac{R B}{0.002 \mathrm{pc} \mathrm{G}}\right)$ & direction \\
\hline galactic wind & \multicolumn{2}{|c|}{$3 \times 10^{17} \mathrm{eV} Z\left(\frac{u}{600 \mathrm{kms}^{-1}}\right)^{2}\left(\frac{T_{A}}{1.5 \times 10^{10} \mathrm{y}}\right)\left(\frac{B}{0.1 \mu \mathrm{G}}\right)$} & \\
\hline \multicolumn{4}{|c|}{ mainly $\gamma^{\prime}$ s, some protons and $\nu$ 's } \\
\hline & \multicolumn{2}{|c|}{ maximal energy } & problem \\
\hline TD's & \multicolumn{2}{|c|}{$\gtrsim 10^{24} \mathrm{eV}$} & exotic \\
\hline
\end{tabular}

Table 1: Maximal energies and problems with the options discussed in this paper. 


\section{Figure Captions}

Figure 1A and 1B: Distance of source versus source energy for protons of arrival energies of $E_{0}=1.7 \times 10^{20} \mathrm{eV}$ and $3.2 \times 10^{20} \mathrm{eV}$, respectively. Plotted from bottom to top are the average distances and the maximal distances at the $1 \sigma, 2 \sigma$ and $3 \sigma$ level, respectively.

Figure 2: Arrival directions of the highest energy events seen by Fly's Eye and the Yakutsk experiment in galactic coordinates [59]. This plot is centered around the galactic anticenter with the middle horizontal line being the projection of the galactic plane. Also shown are nearby galaxy clusters (big circles), AGN's (small circles) and galactic supernova remnants (light circles). As discussed in section 3.3 the galactic magnetic field is supposed to have a coherent component near the galactic plane which in the outer region is polarized in the direction of decreasing galactic longitude. Therefore, the apparent arrival direction of cosmic

rays coming from one of the objects shown should be shifted by $\sim 1.5^{\circ} Z\left(3 \times 10^{20} \mathrm{eV} / E\right)$ to lower latitude. 
This figure "fig1-1.png" is available in "png" format from: http://arxiv.org/ps/astro-ph/9403039v1 
This figure "fig1-2.png" is available in "png" format from: http://arxiv.org/ps/astro-ph/9403039v1 
This figure "fig1-3.png" is available in "png" format from: http://arxiv.org/ps/astro-ph/9403039v1 